\title{
An Empirical Study on Rule Granularity and Unification Interleaving Toward an Efficient Unification-Based Parsing System
}

\author{
Masaaki NAGATA \\ ATR Interpreting T'elephony Research Laboratories \\ 2-2 Hikaridai, Seika-cho, Soraku-gun, Kyoto 619-02, JAl’AN \\ nagala@atr-la.atr.co.jp
}

\begin{abstract}
'I'his paper describes an empirical study on the optimal granularity of the phrase structure rules and the optimal strategy for interleaving $\mathrm{CFG}$ parsing with unification in order to implenent an efficient unification-based parsing system. We clain that using "medium-grained" CHG phrase structure rules, which balance the computational cost of CHC parsing and unification, are a cost-effective solution for rnaking unification-based grammar both eflicient and easy to maintain. We also claim that "late unification", which delays unification until a complete CFG parse is found, saves unnecessary copies of DAGs for irrelevant subparses and improves performance significantly. The effectiveness of these inethods was proved in an extensive experiment. The results show that, on average, the proposed system parses 3.5 times faster than our previous one. The grammar and the parser described in this paper are fully implemented and used as the Japanese analysis module in SL-TRANS, the speech-to-speech translation system of N'llt.
\end{abstract}

\section{Introduction}

Unification-based framework has been an area of active research in natural language processing. Inification, which is the primary operation of this frame. work, provides a kind of constraint-checking mechanism for merging various information sources, such as syntax, semantics, and pragmatics. 'The cornutia tional inefficiency of unification, however, precludes the development of large practical NLP systems, although the framework has many attrictive theoretical properties.

'The efforts made to improve the efliciency of a unification-based parsing system can be classified into four categories.

- CPG parsing algorithra

- Graph unification algorithm

- Grammar representation and organization

- Interaction between CFG parsing and unification
There have been well-known eflicient CFG parsing algorithms such as CKY [Aho and Vllman, 77], Earley [Earley, 70], CHART [Kay, 80], and I,R [Aho and Ullman, 77] ['Iomita, 86]. 'There have also been several recent in-depth studies into efficient graph unification algorithms, whose main concerus have been either avoiding irrelevant copies of 1)AGs [Karttunen and Kay, 85] [Pereira, 85] [Karttunen, 86] [Wroblewski, 87] [Godden, 90] [Kogure, 90] [Tomabechi, 91] [Linele, 91], or the exhaustive expansion of disjunctions into their disjunctive normal forms [Kasper, 87] [Hisele and 1)öre, 88] [Maxwell and Kaplan, 89] [Dörre and Eisele, 90] [Carter, 90] [Nakano, 91].

T'here has, however, been little discussion regarding the optimal representation of a grammar, or linguistic knowledge, in the unification-based framework, from the engineering point of view. Grammar organization is highly flexible, as the unification-based framework uses two different forms of knowledge rep. resentation; atomic phrase structure rules and feature structure descriptions. Method selection greatly af fects both the computational efficiency and the main tenance cost of the system. There has also been little discussion regarding optimal interaction between the CFG parsing process and the unification process in unification-based parsing, which also greatly affects overall performance.

Ilere we int,roduce the notion of granularily, and suggest medium-grained phrase structure rules, in which morpho-syntactic specifications in the feature descriptions are expanded into phrase structure rules. We claim that it reduce the computational loads of unification without intractably increasing the number of rules, and it is optimal in the sense that it satisfies both efficiency and maintainability. Wo also suggest late unification as another solution to the copying problem, as it avoids unnecessary copies of ircelevant subparses by delaying mification until a complete Clici parse is found.

In the following sections, the design and implententalion of the medinum-grained phrase structure rules is explained, then the implementation of the late uni fication is illustrated, and finally the effectivencss of the proposed nethods is proven in experiments. 


\begin{tabular}{|c|c|c|c|}
\hline $\begin{array}{c}\text { Granularity of Phrase } \\
\text { Structure Rules }\end{array}$ & $\begin{array}{c}\text { Consiraints in Phrase } \\
\text { Structure Rules }\end{array}$ & $\begin{array}{c}\text { Constraints in } \\
\text { Feature Descriptions }\end{array}$ & $\begin{array}{c}\text { Number of Phrase } \\
\text { Structure Rules }\end{array}$ \\
\hline Extremely-Coarse-Grained & weak & very strong & $1 \sim 10$ \\
\hline Coarse-Grained & medium & strong & $10 \sim 100$ \\
\hline Medium-Grained & strong & medium & $100 \sim 1000$ \\
\hline Fine-Grained & very strong & weak & $1000 \sim$ \\
\hline
\end{tabular}

Table 1: Granularity of phrase structure rules characterized by the number of rules and the strength of linguistic constraints in the phrase structure rules and the feature descriptions

\section{The Granularity of Phrase Structure Rules}

\subsection{Granularity}

Phrase structure rule granularity has been introduced to refer to the amount of linguistic constraints specified in the atomic CFG phrase structures rules without annotations. 'The rule granularity spectrum has been classified into four categories as shown in Table 1, using the number of grammar rules as a measure.

Unification-based grammars, in general, are characterized by a few general annotated phrase structure rules, and a lexicon with specific linguistic descriptions. This is especially true for HPSG [Pollard and Sag, 87] and JPSG [Gunji, 87], which are to be categorized as extremely-coarse grained, as they drastically reduce the number of phrase structure rules into two for English and one for Japanese, respectively. In these frameworks, the only role of the phrase structure rules is to provide a device for combining a head with its complement. Most linguistic constraints are stored in the feature descriptions.

Coarse-grained rules have been characterized as a grammar consisting of atomic phrase structure rules with medium constraints, and feature descriptions with strong constraints. Medium-grained rules have been characterized as a grammar consisting of atomic phrase structure rules with strong constraints, and feature descriptions with medium constraints. Mediurn-grained rules differ from coarsegrained rules in that they include morpho-syntax in the phrase structure rules, while coarse-grained rules include them in the feature descriptions. This means that medium-grained rules are strong enough to derive syntactic structures from atomic plrase structure rules without feature descriptions.

Grammars for conventional NL.P systems using simple or augmented CFG fall into the category of fine-grained rules, which represent most of linguistic constraints as CFG phrase structure rules, and the number of rules usually amounts to an intractable number of several thousands for practical applications.

\subsection{Maintainability and Efficiency}

In unification-based framework, a linguistic constraint can either be described as atomic context-free phrase structure rules, or as feature descriptions in annotations and lexical entries. As the number of atomic phrase structure rules decreases, the number of feature descriptions increases.

It is true that the lexico-syntactic approach makes the grammar modular and improves its maintainability by reducing the number of rules. However, it must be noted that the computational cost of disjunctive feature structure unification, in the worst case, is exponential in the number of disjunctions [Kasper, 87], whereas the cost of CFG parsing is $o\left(N^{3}\right)$ in the input length $N$. Therefore, extreme rule reduction results in inefficiency. This overwhelms the benefits of the maintainability of the reduced number of rules since grammar development is essentially a trial-and-error process and requires a short turn-around time. However, the cost for CFG parsing also increases as the number of rules increases. Therefore, we must chose the granularity so that the reduction in unification cost outweighs the increase in CFG parsing cost, in order to gain overall efficiency.

\section{The HPSG-Based Japanese Grammars}

In this section, we illustrate the difference between "coarse-grained" rules and "medium-grained" rules using our HPSG-based spoken-style Japanese grammars as an example.

We have developed two unification-based grammars with different granularity ${ }^{1}$, which are essentially based on HPSG and its application to Japanese (JPSG), for the analysis module [Nagata and Kogure, $90]$ of an experimental Japanese-to-English speech-tospeech translation system (SI-TRANS) [Morimoto et a). 900 .

We have selected the "secretarial service of an international conference registration" as our task domain, in which a conversation between a secretary and a questioner is carried out. The Japanese grammars, however, are not task-specific, but rather generalpurpose ones, which cover a wide range of phenom-

\footnotetext{
${ }^{1}$ Historically speaking, we first developed coarse-grained rules and then we mamally transformed them into mediumgrained rules for efficiency.
} 
ena at many linguistic levels from syntax, and semantics, to pragmatics using typed feature structure descriptions. The linguistic phenomena covered in these grammars include:

- Fundamental Constructions: causative, passive, benefactive, negation, interrogative, etc.,

- Control and Gaps: subject/object control,

- Unbounded Dependencies: topic, relative,

- Word Order Variation and Ellipsis.

\subsection{Coarse-Grained Rules Medium-Grained Rules}

Vs.

The coarse-grained HPSG-based Japanese grammar has about 20 generalized phrase structure rules, while the medium-grained grammar has about 200 phrase structure rules. Both grammars use the same lexicon with a vocabulary of about $400 .^{2}$

In the coarse-grained grammar, phrase structure rules only refer to the relative position between the five basic syntactic categories for Japanese: verb ( $V$ ), noun $(N)$, adverb $(A D V)$, postposition $(P)$, and attributive (ATT). Most of the specific linguistic information is encoded as feature descriptions in either the annotation of the phrase structure rules or the lexical entries. In principle, there is no distinction as to whether a constituent is lexical or phrasal, and no subcategories of the 5 basic categories. This contributes greatly to the reduction in the number of phrase structure rules, which results in better grammar maintainability. We present all the plirase structure rules of the coarse-grained Japanese grammar in Appendix $A$.

It has been noticed that the extensive use of disjunctions in feature descriptions, which results from the reduction of the number of phrase structure rules, is the main cause of inefficiency in the coarse-grained version of the grammar. The three major sources of disjunctions are, morpho-syntactic specifications for diverse expressions in the final part of the sentence, free word order and ellipsis of verb complements (subcat slash scrambling), and semantic interpretation of deep case and aspect, where the first two particularly are the problems in spoken-style Japanese.

We have manually converted the coarse-grained phrase structure rules into medium-grained rules to reduce the computational cost of unification. First, we divided each of the basic categories into several subcategories. Then, we divided the coarse-grained phrase structure rules according to the subcategories. To keep the grammar readable, however, we choose to teave the subcat slash scrambling and the semantic

\footnotetext{
${ }^{2}$ We also have another version of the grammal for the same: subcorpus, which is used for the continuous speech recognition module ['Takezawa et al, 91]. It only uses atonic CliG rules, and the number of rules amounts to nore than 2,000 . lt is, therefore, categorized as a fine-grainw grammar in our definition.
}

interpretation undone, and made extensive afforts to expand the mompho-syntibctic specifications.

\subsection{Example: Medium-Grained Rules for Predicate Verb Phrases}

In this section, we illustrate the process of transformation using a predicate verb plurase production rule as an example. Japanese predicate phrases consist of a main verb followed by a sequence of auxiliaries and sentence final particles. 'There is an almost onedimensional order of verbal constituents such as in Figure 1, which reflects the basic hierarchy of the Japarese sentence structure.

Kernel verbs occur first in a predicate phrase scquence. Voice auxiliaries precede all other auxiliaries, and within this category, the causative auxiliary (sa)seru precedes the passive auxiliary (ra)rett. Aspect auxiliaries, such as the progressive auxiliary (te)iru precede modal auxiliaries and follow voice auxiliaries. Modal auxiliaries are classified into two groups with respect to the relative order of negative and tense auxiliaries. Moodl includes the optative auxiliaries, such as tai (want), beki (should/must), etc. Mood2 includes the evidential or inferential auxiliaries such as rashii (seem/look), kamoshirenai (may), etc. Negative auxiliaries nai, n (not) follow voice, uspect, and mood 1 auxiliaries, and precede tense and mood2 auxiliaries. Tense auxiliaries $t a$, da (-ed) show irregular behavior. 'They follow the voice, aspect, mood1, and negative auxiliaries, and precede the mood2 auxiliaries. 'They also can follow the mood 2 auxiliaries.

In the coarse-grained grammar, we provide a single plirase structure rule for the phenomena.

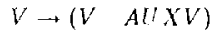

The order constraints between auxiliaries are specified in the annotation of rule (1) and each lexical entry by the combination of the syntactic fea tures, such as the syn/head/subcat for preceding constituente, the syn/head/coh for following constituents, and the synthead/modi for the position of the con stituents in the verb phrase hierarchy. For example, the causative auxiliary verb sert has the following, feature bundles in its synfitiead/modl feature.

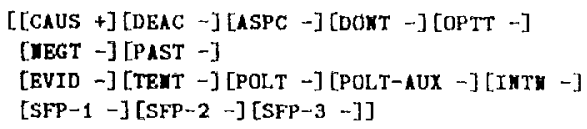

In converting the rule, first we have classified the verbal phrasal categories according to the hierar. chy, e.g. V-kermel, V-aspect, V-mood1, V-negt, Vmood2, and $V$-tense, then we have subcategorized the auxiliaries as shown in Table 2. 'Thus, the coarsegrained phrase structure rule (1) is converted to the 32 medium-grained grammar tules in Appendix $B$. 


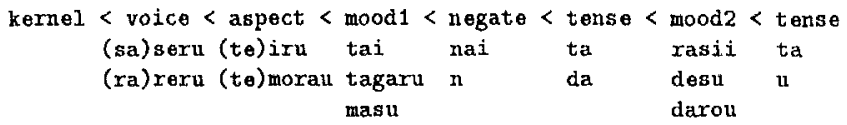

Figure 1: The predicate hierarchy of Japanese

\begin{tabular}{|l|l|}
\hline AUXV-caus & causative auxiliary: (sa)seru \\
\hline AUXV-deac & passive auxiliary: (ra)reru \\
\hline AUXV-aspc & aspect auxiliary: (te)iru, (te)aru \\
\hline AUXV-dont & benefactive auxiliary: (te) morau \\
\hline AUXV-optt & optative auxiliary: tai, beki \\
\hline AUXV-negt & negative auxiliary: nai, $n$ \\
\hline AUXV-tense & tense auxiliary: ta, da \\
\hline AUXV-evid & evidential auxiliary: rashii, darou \\
\hline AUXV-copl & copulative auxiliary: da, desu \\
\hline
\end{tabular}

Table 2: Subcategories of auxiliaries in the mediumgrained grammar

\section{Interleaving CFG Parsing and Unification}

\subsection{Strategies for Evaluating Feature Descriptions}

Unification is an expensive operation, so the point of evaluating feature descriptions during CFG parsing has serious affects on the overall performance. We have implemented two strategies for feature description evaluation:

\section{Early Unification (Step-by-step Strategy)}

Feature descriptions are evaluated step-by-step, at each rule invocation in the CFG parsing

Late Unification (Pipeline Strategy) Feature descriptions are evaluated when a complete CFG parse is found. The "well-formedness" of a parse derived from atomic CFG rules is verified by evaluating associated feature descriptions.

The granularity of the phrase structure rules is closely related to the proper selection of the evaluation strategy. Since the atomic phrase structure rules in the coarse-grained grammar are not so strong as to constrain syntactic structures, we have to employ the early unification to avoid a number of irrelevant subparses which should have been eliminated by the evaluation of annotations. However, since the atomic rules in the medium-grained grammar liave detailed morpho-syntax specifications, they should be able to avoid irrelevant copies by using the late unification.

\subsection{Implementing the Evaluation Strategies}

We have implemented the various evaluation strategies by doing additional housekeeping in the underlying parser. The parser used here is called the Typed

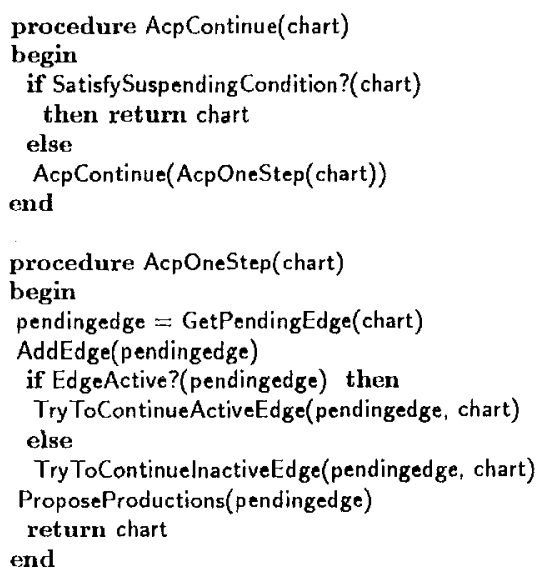

Figure 2: Iterative Rule Invocation in an Active Chart Parsing Algorithm

Feature Structure Propagation Parser (TFSP Parser) [Kogure, 89], which is based on the active chart parsing algorithm [Kay, 80] and typed feature structure unification [Ä̈t-Kaci, 86].

The active chart parser and the unification algorithm are implemented in $\mathrm{C}$ on Sun 4, which is a 10-MIPS work station. 'The unification algorithm is based on nondestructive graph unification [Wroblewski, 87], which we extend to treat negation, loop, type symbol subsumption relationships, and disjunction. Successive approximation [Kasper, 87] is used for disjunctive feature structure unification.

The Active chart parsing algorithm basically consists of chart initialization and iterative rule invocation. The basic part of the iterative rule invocation is shown in Figure 2. AcpContinue checks the suspending condition and calls rule invocation recursively. AcpOneStep carries out a cycle of rule invocation which consists of getting a new pending edge (GetPendingEdge), adding it to the chart (AddEdge), combining active and inactive edges (TryToContinueActiveEdge/TryToContinuelnactiveEdge), and proposing new edges (ProposeProductions). The parser stops (SatisfySuspendingCondition?) when it finds an inactive edge whose starting and ending vertex are the left-most and right-most vertex of the chart, respectively, and whose label is the start symbol of the grammar. 
In early unification, the feature descriptions are evaluated when the edges are combined, while in late unification, they are evaluated in the chart suspending condition clieck only if the chart suspending condition holds. Delaying unification is implemented by adding a slot edge.parse to the edge structure, which keeps a list of the pair of active and inactive edges constructing the edge. If either or both of the argument feature structures of the unification have not been evaluated, they are recursively evaluated to get the target feature structure.

It has to be noted that some derivations that ter minate when feature descriptions are evaluated, may not terminate if they are ignored. For example, it is possible to write a rule for unbounded dependency like (2), in which an element in the subcat feature is moved to the slash feature, to introduce slashed categories dynamically ${ }^{3}$.

$$
\alpha \rightarrow(\alpha)
$$

Ignoring feature descriptions in the rule may cause an infinite loop. Therefore, feature descriptions are forced to be evaluated, when rules that cause a loop are encountered in late unification.

\section{Experiment}

The effectiveness of the strategies proposed in this paper can be judged by observing their behavior in practice. We have tested the time behavior of parsing with respect to rule granularity and interleaving strategy of $\mathrm{CFG}$ parsing and unification. 85 sanple sentences are used. 'These are selected from the sample subcorpus of ATR's dialogue corpus whose task domain is the "secretarial service of an international conference". The average length of the sample sentences is 11.0 characters, and their maximum and minimum length are 2 and 28 characters, respectively.

We have developed two Japauese grammars of different gramlarity with almost the same coverage. The conse-grained rules consist of 22 generalized phrase structure rules with detailed teature (lescription in their annotations, while the mediun yrained rules consist of 164 detailed phrase structure rules with less detailed feature descriptions. Both grammars use the sane lexicon with about 400 lexical entries. We have also implemented two different feature description evaluation modes in the active chart. parser. 'The early unification evaluation mode cvaluates the feature descriptions at each rule application (the step-by-step strategy). The late unification cvaluation mode, on the other hand, delays unification until a complete symtactic structure is found by using the atomic phrase structure rules only (the pipeline? strategy).

'The average parsing time is shown in Tatble 3 . It. shows that, on average, the medium-gramed gratu-

\footnotetext{
${ }^{3}$ In our implementation, for efliciency reasons, we: genwerot all the appropriate combinations of subcat and slash in torlvance, and keep them as a disjunctive feature structure
}

\begin{tabular}{|c|c|c|c|}
\hline Rule Granularity & Course & Medium & Medium \\
\hline Unification Mode & Harly & Carly & Iate \\
\hline Average lkuntime & $30.2 \mathrm{sec}$ & $17.8 \mathrm{sec}$ & $8.7 \mathrm{sec}$ \\
\hline Relative Specd & 1.0 & 1.7 & 3.5 \\
\hline
\end{tabular}

Table 3: Average parsing time with respect to granularity and unification mode

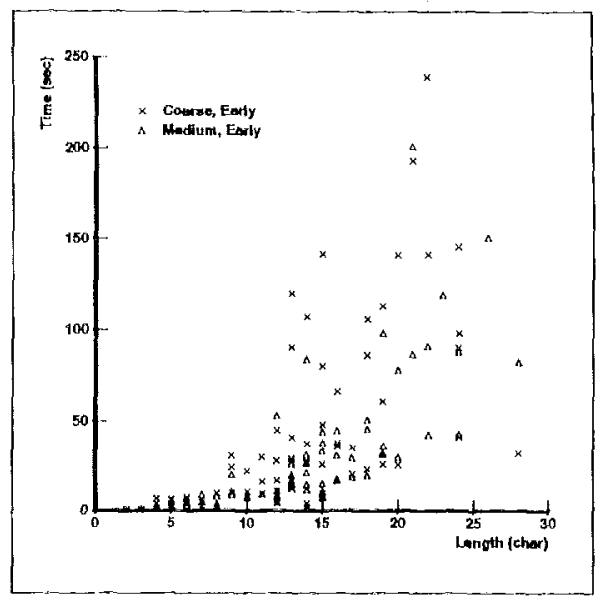

Figure 3: Comparison of Coarse-Grained Rules and Medium-Grained Rules

mar rules are 1.7 times more efficient than the coarsegrained rules in the early unification mode, and that the late unification node is 2.0 times more efficient. than the early unilication mode with the mediumgrained grammar. Moreover, when the mediumgrained grammar rules and the late unification mode ar' combined, the new parser runs 3.5 times faster than the previous one using the coarsograined grammar rules and the early unification.4

\section{Discussion}

The relationship, between input length and parsing time with respect to grammar granularity is shown in Figure 3. In gencral, the mediun-grained rules performed better than the coarse-graned rules. 'This temdency becomes clearer, as the sentence length increases. This rosults from the reduction of disjunc. tive feature descriptions whose computational cost in-

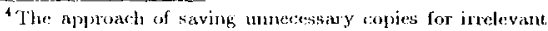
subparses in a parsing process by late mification is or thogomal

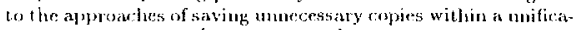
lim process, such as [Tomitwechi, ?h] . Therefore, the effects of sperel up can be multiplied. We have alrearly implemented his cuasi-destructive glaph unification, and the prelininary t:xperinent resule shews that thr parser with new unifier roms alment. 1 whe ds fast ets the one treported in this paper.
} 


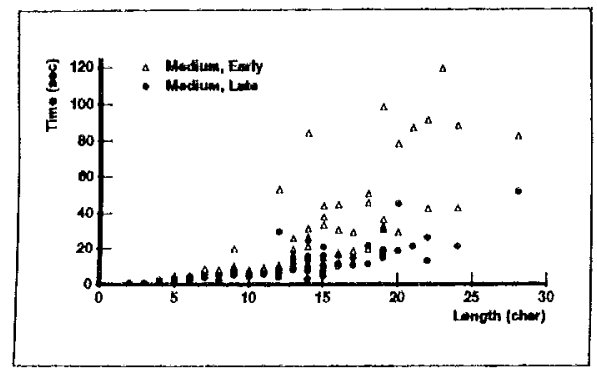

Figure 4: Comparison of Farly Unification and Late Unification

creases exponentially in the number of disjunctions. However, we occasionally encounter sentences which are parsed faster using coarse-grained rules rather than medium-grained rules. This is because the increase in the atomic CFG parsing cost exceeds the reduction of the unification cost.

The relationship between input length and parsing time with respect to unification evaluation mode is shown in Figure 4. This shows that the late unification mode is significantly more efficient than the early unification mode. It also shows that the parsing time in the late unification mode seems to be polynomial (not exponential) in the input length, while that in the early unification mode varies widely and irregularly. This is because the parsing time in the late unification mode is mainly predominated by the cost of atomic CFG parsing by delaying unification, whereas the parsing time in the early unification mode is mainly predominated by the cost of unification.

We have demonstrated the effectiveness of combining medium-grained phrase structure rules with late unification. Experiment results suggest that new prospective techniques for speeding up unificationbased parsing exist.

The first is automatic transformation of phrase structure rules, which converts disjunctions in the feature descriptions in to atomic phrase structure rules. Some disjunctions such as subcat slash scrambling are so regular that it seems possible to expand them into a set of CFG rules using formal procedures. If the grammar compiler can perform this kind of transformation automatically, wo can gain efficiency without losing grammar maintainability.

The second is feature-sensitive lazy unification. Unification is used for both building up a structure using information-propagation and blocking rule application using constraint-checking. If the grammar compiler can separately output those features for constraint-checking such as syntactic features, and those for information-propagation such as semantic representations, irrelevant subparses can be pruned efficiently by evaluating the constraint-checking features first. Unification is an associative and commu- tative operation, so the same results from the featuresensitive lazy unification are assured.

The third is parallel implementation of a unification-based parser based on late unification (pipe-line strategy). In early unification (step-by-step strategy), it is hard to perform parsing in paraliel because the CFG parsing process and the unification process depend strongly on each other. However, both processes are completely separated in the pipe-line strategy. Therefore, it is easy to introduce the existing parallel algorithms to both CFG parsing and unification. It is estimated that most feature descriptions can be evaluated in parallel, at least, at the lexical level, because unification-based grammars such as HPSG derive phrase structure by iteratively propagating the local constraints.

\section{Conclusion}

In this paper, we have proposed two techniques for implementing an efficient unification-based parsing system, which, when combined, significantly improve the overall performance. The first is changing the granularity of the context-free phrase structure rules into medium-grained rules. This enables us to reduce the amount of unification for feature descriptions without intractably increasing the number of phrase structure rules. The second is late unification in which the unification for feature descriptions is delayed until a complete CFG parse is found. 'This saves unnecessary copies of feature structures which are wasted for irrelevarit subparses.

We have tested the time behavior of the parsing system using two grammars of different granularity (coarse/medium) and two different strategies for invoking unification (early/late). It is proved that, on average, late unification using medium-grained rules parses 3.5 times faster than the previous early mification using coarse-grained rules.

\section{Acknowledgments}

T'he author would like to thank Dr. Kurenatsu, and all the nembers of AT'R Interpeting Telephony Research l,als. for their constant help and fruitful discussions.

\section{References}

[Aho and Ulnnan, 77] Aho, A. and Ullman, J., Principles of Compiler Design Addison-Wesley, 1977.

[Ait-Kaci, 86] Nit-Kaci, H., "An Algebraic Semantics $\Lambda_{p-}$ proach to the Effective Resolution of Type Fquations," Journal of Theoretical Compuler Science 45, 1986.

[Carter, 90] Carter, D., "Eflicient Disjunctive Unification for Bottom-Up Parsing," Proc. of COLING-00, 1990.

[Döre and Fisele, 90] Döre and Visele, "Feature Logic with Disjunctive Unification," Iroc. of COLING-10, 1900.

[Warley, 70] Earley, J., "An Ffficient Context-Fee Parsing Al goritlm," $A C M, 19,2,1970$.

[Eisele and D)örre, 88] Eiscle, A. and Dörc, J., "Unification of Disjunctive Feature Descriptions," Proc. of the $26 \mathrm{Lh} A C L$, 1988. 
[Emele, 91] Emele, M., "Unification with Lazy Non-Hedumdant Copying," Proc. of the e9th $A C I_{1} 1991$.

[Godden, 90] Godden, K., "I,azy Unification," Proc. of the 28th ACL, 1990.

[Gunji, 87] Gunji, J., Japanese Phrase Structure Grammar A Unification-Based Approach, Dortrecht, Heidel, 1987.

[Kasyer, 87] Kasper, H., "A Unification Method for Disjunctive Feature Descriptions," Proc. of the 25 th ACL, 1987.

[Karttunen, 86] Karttunejt, L., D-PATI $-\Lambda$ I) vironment for Unification-Based Grammars, CSLI-86-91, CSLI, 1986.

[Karttunen and Kay, 85] Karttunen, L. and Kay, M., "Structure Shaxing with Binary Trees," Proc. of the 29 rd $A C L$, 1985.

[Kay, 80] Kay, M., Algorithm Scheriata and Hata Structures in Syntactic Processing, Tednucal Feport CS1 8 80-12, Xerox PARC, 1980.

[Kogure, 89] Kogure, K., "Parsing Jrprovese Spoken Sentences based on IIPSG," Proce of the IWI'I', 1989.

[Kogure, 90] Kogure, K., "Strategic Laxy Incremental Copy Graph Unification," Proc. of COLING-90, 1990.

[Maxwell and Kaplan, 89] Maxwell, J. and Kaplar, L., "An Overview of Disjunctive Constrajnt Satisfaction," Prot. of the IWPT, 1989.

[Morimoto et al, , 90] Morimoto,T. et al., "Integration of Speech lecognition and Language P'rocessing in Spoken Language Tranglation System (SI-TRANS)," Proc. of the ICSISP, 1990.

[Nagata and Kogure, 90] Nagata, M. and Kogure, K., "HPSG-Based lattice Parser for Spoken Japaruese in a Spoken I,anguage Translation System," Proc. of the 9th LCAI, 1990.

[Naknno, 91] Naknno, M., "Constraint Projection: An lifficient 'lreatment of Disjunctive Fitature I Jescriptions," I'roc of 29 th $A C H, 1091$.

[Pereira, 85] Pereirn, $F$., "A Stuncturs-Sharing fiepresent tion for Unitication-Hased Formalisms," Proc. of the $29 \mathrm{rd}$ $A C L, 1985$.

[Pollard and Sag, 87] Pollard, C. nnd Sag, I., An InformationHased Syntax and Semantics, CSLI Iecture Notes No. 13, CSSLI, 1987

['Takezawa th al, , 91] Takezawa, 'T', et al., "Linguistic: Constraints for Continuous Speech Recognition in (ivalDirected Dialogue," Proc. of ICASSP-91, 1991.

[Tomabechi, 91] 'I'muabechi, Il., "Quasi-Destructive: Grapph Unification," Proc, of s9th ACL, 1991.

[Tomita, 86] 'Lomita, M., Nfficient Parsing for Natural Language: A Fisi Algorithm for Praclical Systems, Kluwer Acadernic l'ublishers, 1986 .

[Wroblewski, 87] Wroblewski, J)., "Nondestructive (iraph Unification," Proc. of the th $A A A I, 1987$.

\section{Appendix}

\section{A Coarse-grained Grammar Rules for Japanese}

The name of each rule is shown in the comment, where the suffix - ah, -ch, -coord means adjunction, complementalion, and coordination, respectively.

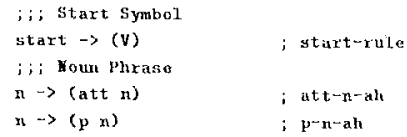

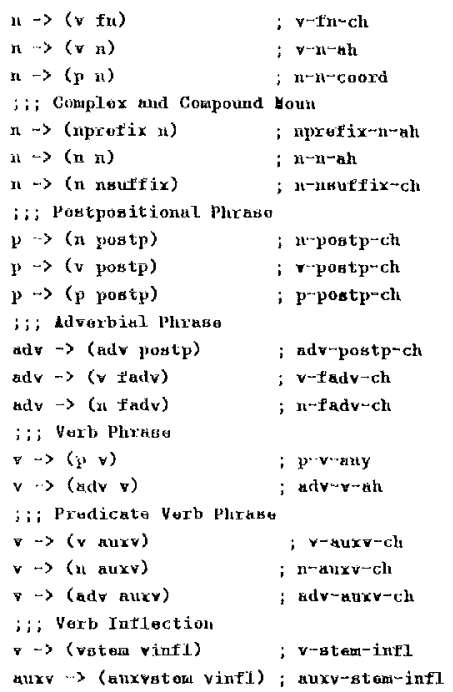

\section{B Medium-grained Rules for Japanese Verb Phrases}

J'he coarse. grained grammar rule (1) is converted to the following 32 modium-gi ained grammar rules.

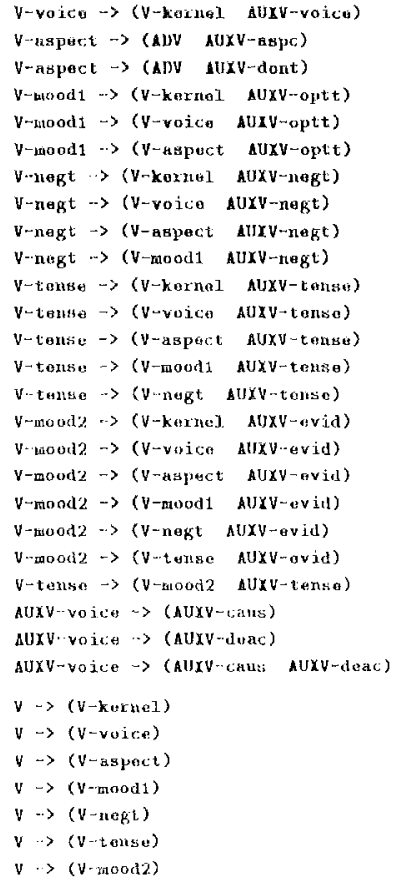

